\title{
$(2+1)$-Dimensional Duffin-Kemmer-Petiau Oscillator under a Magnetic Field in the Presence of a Minimal Length in the Noncommutative Space
}

\author{
Bing-Qian Wang, Zheng-Wen Long, Chao-Yun Long, and Shu-Rui Wu \\ College of Physics, Guizhou University, Guiyang 550025, China \\ Correspondence should be addressed to Zheng-Wen Long; zwlong@gzu.edu.cn
}

Received 17 March 2017; Accepted 11 May 2017; Published 18 July 2017

Academic Editor: Sally Seidel

Copyright (C) 2017 Bing-Qian Wang et al. This is an open access article distributed under the Creative Commons Attribution License, which permits unrestricted use, distribution, and reproduction in any medium, provided the original work is properly cited. The publication of this article was funded by SCOAP . $^{3}$

Using the momentum space representation, we study the $(2+1)$-dimensional Duffin-Kemmer-Petiau oscillator for spin 0 particle under a magnetic field in the presence of a minimal length in the noncommutative space. The explicit form of energy eigenvalues is found, and the wave functions and the corresponding probability density are reported in terms of the Jacobi polynomials. Additionally, we also discuss the special cases and depict the corresponding numerical results.

\section{Introduction}

The interest in the study of the minimal length uncertainty relation combination with the noncommutative space commutation relations in nonrelativistic wave equation and relativistic wave equation has drawn much attention $[1,2]$ in recent years. Motivated by string theory, loop quantum gravity, and quantum geometry [3-15], the modification of the ordinary uncertainty relation has become an appealing case of research. In the so-called minimal length formulation, Kempf and Hinrichsen [16-18] have shown that the minimal length can be introduced as an additional uncertainty in position measurement, so that the usual canonical commutation relation between position and momentum operator is substituted by $[x, p]=i \hbar\left(1+\beta p^{2}\right)$, where $\beta$ is a small positive parameter called the deformation parameter. Recently, various topics have been studied in connection with the minimal length uncertainty relation [19-28]. On the other hand, the issue of noncommutative (NC) quantum mechanics has also been extensively discussed. The noncommutativity of space-time coordinates was first introduced by Snyder [29] aiming to improve the problem of infinite selfenergy in quantum field theory, and the noncommutative geometry has been put forward because of the discovery in string theory and matrix model of M-theory [30]. Recently, various aspects of both NC classical [31] and quantum [32] mechanics have been extensively studied devoted to exploring the role of NC parameter in the physical observables [33-40]. Recently, the effect of the quantum gravity on the quantum mechanics by modifying the basic commutators among the canonical variables has been an attractive topic; therefore the combination of the minimal length uncertainty relation and the noncommutative space commutation relations is a colorful problem. In the present work, we are interested to study the two sectors in the framework of the relativistic DKP equation and analyze the effects of them on the energy spectrum and the corresponding wave functions.

The organization of this work is as follows. In Section 2, we consider the DKP oscillator for spin 0 particle in the presence of a minimal length in the NC space. In Section 3, we study the problem under a magnetic field. In Section 4, we discuss some special cases of the solutions to check the validity of our results. Finally, the work is summarized in last section. 


\section{The $(2+1)$-DKP Oscillator for Spin 0 Particle in the Presence of the Minimal Length in NC Space}

In NC space, the canonical variables satisfy the following commutation relations:

$$
\begin{aligned}
& {\left[x_{i}^{(\mathrm{NC})}, x_{j}^{(\mathrm{NC})}\right]=i \theta_{i j},} \\
& {\left[P_{i}^{(\mathrm{NC})}, P_{j}^{(\mathrm{NC})}\right]=0,} \\
& {\left[x_{i}^{(\mathrm{NC})}, P_{j}^{(\mathrm{NC})}\right]=i \hbar \delta_{i j},}
\end{aligned}
$$

$$
(i, j=1,2)
$$

where $\theta_{i j}=\epsilon_{i j} \theta$ is the antisymmetric NC parameter, representing the noncommutativity of the space, and $x_{i}^{(\mathrm{NC})}$ and $P_{i}^{(\mathrm{NC})}$ are the coordinate and momentum operators in the NC space. By replacing the normal product with star product, the DKP equation in commutation space will change into the DKP equation in NC space as

$$
H(P, x) * \psi(x)=E \psi(x) .
$$

Usually, one way to deal with the problem of NC space is via the star product or Moyal-Weyl product on the commutative space functions:

$$
(f * g)(x)=\left.\exp \left[\frac{i}{2} \theta_{i j} \partial_{i}^{x} \partial_{j}^{y}\right] f(x) g(y)\right|_{y \rightarrow x},
$$

where $f(x)$ and $g(y)$ are arbitrary infinitely differentiable functions.

Then the Moyal-Weyl product can be replaced by a Bopp shift of the form

$$
\begin{aligned}
& x_{i}^{(\mathrm{NC})}=\widehat{x}_{i}-\frac{1}{2 \hbar} \theta_{i j} \widehat{P}_{j}, \\
& P_{i}^{(\mathrm{NC})}=\widehat{P}_{i},
\end{aligned}
$$

and therefore in the two-dimensional NC space, (4) can be expressed as

$$
\begin{aligned}
& x^{(\mathrm{NC})}=\widehat{x}-\frac{\theta}{2 \hbar} \widehat{P}_{y}, \\
& y^{(\mathrm{NC})}=\widehat{y}+\frac{\theta}{2 \hbar} \widehat{P}_{x}, \\
& P_{x}^{(\mathrm{NC})}=\widehat{P}_{x}, \\
& P_{y}^{(\mathrm{NC})}=\widehat{P}_{y},
\end{aligned}
$$

where $\hat{x}, \widehat{y}, \widehat{P}_{x}$, and $\widehat{P}_{y}$ are the position and momentum operators in the usual quantum mechanics, respectively, which satisfy the canonical Heisenberg commutation relations.
Thus, the relativistic DKP equation for a free boson of mass $m$ is given by $[41,42]$

$$
\left[c \vec{\beta} \cdot \vec{P}+m c^{2}\right] \psi^{\prime}=i \hbar \beta^{0} \frac{\partial}{\partial t} \psi^{\prime},
$$

where $\vec{\beta}=\left(\beta^{1}, \beta^{2}, \beta^{3}\right)$ and $\beta^{0}$ is the DKP matrices which meet the following algebra relation:

$$
\beta^{\mu} \beta^{v} \beta^{\lambda}+\beta^{\lambda} \beta^{v} \beta^{\mu}=\mathrm{g}^{\mu \nu} \beta^{\lambda}+\mathrm{g}^{\lambda v} \beta^{\mu},
$$

where $\mathrm{g}^{\mu \nu}=\operatorname{diag}(1,-1,-1,-1)$ is the metric tensor in Minkowski space. For spin 0 particle, $\boldsymbol{\beta}^{i}$ are $5 \times 5$ matrices expressed as

$$
\begin{aligned}
& \boldsymbol{\beta}^{0}=\left[\begin{array}{cc}
I_{0} & \overline{0} \\
\overline{0}^{T} & \boldsymbol{\sigma}
\end{array}\right], \\
& \boldsymbol{\beta}^{i}=\left[\begin{array}{cc}
\widehat{0} & k_{i} \\
-\left(k_{i}\right)^{T} & \boldsymbol{\sigma}
\end{array}\right], \quad i=1,2,3,
\end{aligned}
$$

with $\mathbf{\sigma}, \overline{0}$, and $\widehat{0}$ being $3 \times 3,2 \times 3$ and $2 \times 2$ zero matrices, respectively. Other matrices in (8) are given as follows:

$$
\begin{aligned}
& I_{0}=\left[\begin{array}{ll}
0 & 1 \\
1 & 0
\end{array}\right], \\
& k_{1}=\left[\begin{array}{ccc}
-1 & 0 & 0 \\
0 & 0 & 0
\end{array}\right], \\
& k_{2}=\left[\begin{array}{ccc}
0 & -1 & 0 \\
0 & 0 & 0
\end{array}\right], \\
& k_{3}=\left[\begin{array}{ccc}
0 & 0 & -1 \\
0 & 0 & 0
\end{array}\right] .
\end{aligned}
$$

The DKP oscillator is introduced by using substitution of the momentum operator $\vec{P}$ with $\left(\vec{P} \rightarrow \vec{P}-i m \omega \eta^{0} \vec{r}\right)$, where the additional term is linear in $r, w$ is the oscillator frequency, and $\boldsymbol{\eta}^{0}=2\left(\boldsymbol{\beta}^{0}\right)^{2}-I$ with $\left(\boldsymbol{\eta}^{0}\right)^{2}=I$.

It is easy to get the $2 \mathrm{D} \mathrm{DKP}$ oscillator from the above equation:

$$
\left[c \overrightarrow{\boldsymbol{\beta}} \cdot\left(\vec{P}-i m \omega \boldsymbol{\eta}^{0} \vec{r}\right)+m c^{2}\right] \psi=i \hbar \boldsymbol{\beta}^{0} \frac{\partial}{\partial t} \psi
$$

Given this situation above, considering the NC formalism, and via the Bopp shift (4)-(5), the $(2+1)$-dimensional DKP oscillator equation in NC space becomes

$$
\begin{aligned}
& {\left[c \boldsymbol{\beta}^{1}\left(P_{x}^{(\mathrm{NC})}-i m w \boldsymbol{\eta}^{0} x^{(\mathrm{NC})}\right)\right.} \\
& \left.\quad+c \boldsymbol{\beta}^{2}\left(P_{y}^{(\mathrm{NC})}-i m w y^{(\mathrm{NC})}\right)+m c^{2}\right] \psi=\boldsymbol{\beta}^{0} E .
\end{aligned}
$$


For a boson of spin 0 , the spinor $\psi$ is a vector with five components $[43,44]$, which reads

$$
\psi=\left(\psi_{1}, \psi_{2}, \psi_{3}, \psi_{4}, \psi_{5}\right)^{T}
$$

Substituting $\psi$ into (11) we have

$$
\begin{aligned}
& -m c^{2} \psi_{1}+E \psi_{2}+c\left(P_{x}^{(\mathrm{NC})}+i m w x^{(\mathrm{NC})}\right) \psi_{3} \\
& +c\left(P_{y}^{(\mathrm{NC})}+i m w y^{(\mathrm{NC})}\right) \psi_{4}=0, \\
& E \psi_{1}-m c^{2} \psi_{2}=0, \\
& c\left(P_{x}^{(\mathrm{NC})}-i m w x^{(\mathrm{NC})}\right) \psi_{1}+m c^{2} \psi_{3}=0, \\
& c\left(P_{y}^{(\mathrm{NC})}-i m w y^{(\mathrm{NC})}\right) \psi_{1}+m c^{2} \psi_{4}=0, \\
& m c^{2} \psi_{5}=0 .
\end{aligned}
$$

Combination of (13) gives

$$
\begin{gathered}
\left\{\left[\widehat{P}_{x}+i m w\left(\widehat{x}-\frac{\boldsymbol{\theta}}{2 \hbar} \widehat{P}_{y}\right)\right]\left[\widehat{P}_{x}-i m w\left(\widehat{x}-\frac{\boldsymbol{\theta}}{2 \hbar} \widehat{P}_{y}\right)\right]\right. \\
+\left[\widehat{P}_{y}+i m w\left(\widehat{y}+\frac{\boldsymbol{\theta}}{2 \hbar} \widehat{P}_{x}\right)\right] \\
\left.\quad \cdot\left[\widehat{P}_{y}-i m w\left(\widehat{y}+\frac{\boldsymbol{\theta}}{2 \hbar} \widehat{P}_{x}\right)\right]+m^{2} c^{2}-\frac{E^{2}}{c^{2}}\right\} \psi_{1}=0 .
\end{gathered}
$$

In addition, in the minimal length formalism, the Heisenberg algebra is given by

$$
\left[\widehat{x}_{i}, \widehat{P}_{j}\right]=i \hbar \boldsymbol{\delta}_{i j}\left(1+\alpha P^{2}\right)
$$

where $\alpha$ is the minimal length positive parameter. Moreover, in the momentum space, the position vector and momentum vector can be expressed as

$$
\begin{aligned}
& \widehat{x}_{i}=i \hbar\left[\left(1+\alpha P^{2}\right) \frac{\partial}{\partial p_{i}}\right], \\
& \widehat{P}_{i}=P_{i}
\end{aligned}
$$

and substituting (15) and (16) into (14) we have

$$
\begin{aligned}
& {\left[\left(1+\frac{m^{2} w^{2} \boldsymbol{\theta}^{2}}{4 \hbar^{2}}\right)\left(P_{x}^{2}+P_{y}^{2}\right)+m^{2} w^{2}\left(x^{2}+y^{2}\right)\right.} \\
& -2 m w \hbar\left(1+\alpha P^{2}\right)-\frac{m^{2} w^{2} \boldsymbol{\theta}}{\hbar}\left(1+\alpha P^{2}\right) L_{z}+m^{2} c^{2} \\
& \left.-\frac{E^{2}}{c^{2}}\right] \psi_{1}=0 .
\end{aligned}
$$

Now, in order to solve (17), an auxiliary wave function is defined as $\psi_{1}(P, \vartheta)=e^{i \ell \vartheta} \phi(P)$, and for the sake of simplicity, we bring the problem into the polar coordinates, recalling that

$$
\begin{aligned}
P_{x} & =P \cos \vartheta, \\
P_{y} & =P \sin \vartheta \\
\frac{\partial}{\partial P_{x}} & =\cos \vartheta \frac{\partial}{\partial P}-\frac{\sin \vartheta}{P} \frac{\partial}{\partial \vartheta}, \\
\frac{\partial}{\partial P_{y}} & =\sin \vartheta \frac{\partial}{\partial P}+\frac{\cos \vartheta}{P} \frac{\partial}{\partial \vartheta} ;
\end{aligned}
$$

then (17) becomes

$$
\begin{aligned}
& \left\{\left(1+\alpha P^{2}\right)^{2} \frac{\partial^{2}}{\partial P^{2}}\right. \\
& +\left[\frac{1}{P}\left(1+\alpha P^{2}\right)^{2}+2 \alpha P\left(1+\alpha P^{2}\right)\right] \frac{\partial}{\partial P} \\
& -\frac{\ell^{2}}{P^{2}}\left(1+\alpha P^{2}\right)^{2} \\
& +\frac{-\left(1+m^{2} w^{2} \theta^{2} / 4 \hbar^{2}\right)+2 m w \alpha \hbar+m^{2} w^{2} \theta \alpha \ell}{m^{2} w^{2} \hbar^{2}} P^{2} \\
& \left.+\frac{\left(\left(E^{2}-m^{2} c^{4}\right) / c^{2}+2 m w \hbar+m^{2} w^{2} \theta \ell\right)}{m^{2} w^{2} \hbar^{2}}\right\} \phi(P) \\
& =0 ;
\end{aligned}
$$

with the help of a variable transformation

$$
q=\frac{1}{\sqrt{\alpha}} \tan ^{-1}(\sqrt{\alpha} P),
$$

which will map the variable $P \in(0, \infty)$ to $q \in(0, \pi / 2 \sqrt{\alpha})$, we simplify (19) to

$$
\begin{aligned}
& {\left[\frac{\partial^{2}}{\partial q^{2}}+\left(\frac{v}{\mu}+\frac{\mu}{v}\right) \sqrt{\alpha} \frac{\partial}{\partial q}-\alpha \ell^{2} \frac{v^{2}}{\mu^{2}}+\alpha k^{\prime} \frac{\mu^{2}}{v^{2}}+\alpha \varepsilon^{\prime}\right]} \\
& \cdot \phi(q)=0,
\end{aligned}
$$


where

$$
\begin{aligned}
\mu= & \sin (\sqrt{\alpha} q), \\
v= & \cos (\sqrt{\alpha} q), \\
k^{\prime}=- & \boldsymbol{\ell}^{2} \\
& +\frac{-\left(1+m^{2} w^{2} \boldsymbol{\theta}^{2} / 4 \hbar^{2}\right)+2 m w \alpha \hbar+m^{2} w^{2} \boldsymbol{\theta} \alpha \boldsymbol{\ell}}{\alpha^{2} m^{2} w^{2} \hbar^{2}}, \\
\mathcal{\varepsilon}^{\prime}= & \frac{\left(E^{2}-m^{2} c^{4}\right) / c^{2}+2 m w \hbar+m^{2} w^{2} \boldsymbol{\theta} \boldsymbol{\ell}}{\alpha m^{2} \boldsymbol{\omega}^{2} \hbar^{2}}-2 \boldsymbol{\ell}^{2} .
\end{aligned}
$$

Next, for convenience, another auxiliary function is introduced by $\phi(q)=v^{\lambda} F(q)$, with $\lambda$ being a constant to be determined. Thus we have

$$
\begin{aligned}
& \left\{\frac{\partial^{2}}{\partial q^{2}}+\left(\frac{v}{\boldsymbol{\mu}}+(1-2 \lambda) \frac{\boldsymbol{\mu}}{v}\right) \sqrt{\alpha} \frac{\partial}{\partial q}-\alpha \boldsymbol{\ell}^{2} \frac{v^{2}}{\boldsymbol{\mu}^{2}}\right. \\
& \left.\quad+\alpha\left[k^{\prime}+\lambda(\lambda-1)-\lambda\right] \frac{\boldsymbol{\mu}^{2}}{v^{2}}+\alpha\left(\varepsilon^{\prime}-2 \lambda\right)\right\} F(q) \\
& \quad=0 .
\end{aligned}
$$

Here, in order to simplify above mathematical expression, we select $k^{\prime}+\lambda(\lambda-1)-\lambda=0$; then it leads to the following expression of $\lambda$ :

$$
\begin{aligned}
& \lambda=1+\sqrt{1-k^{\prime}}, \\
& \lambda^{\prime}=1-\sqrt{1-k^{\prime}} .
\end{aligned}
$$

Since the second solution leads to a nonphysically acceptable wave function, then (23) turns into

$$
\begin{aligned}
& {\left[\frac{\partial^{2}}{\partial q^{2}}+\left(\frac{v}{\mu}+(1-2 \lambda) \frac{\mu}{v}\right) \sqrt{\alpha} \frac{\partial}{\partial q}-\alpha \ell^{2} \frac{v^{2}}{\mu^{2}}\right.} \\
& \left.+\alpha\left(\varepsilon^{\prime}-2 \lambda\right)\right] F(q)=0 .
\end{aligned}
$$

Then with the help of another auxiliary function $F(q)=$ $\mu^{\ell} \zeta(q)$, thus (25) reads

$$
\begin{aligned}
& \left\{\frac{\partial^{2}}{\partial q^{2}}+\left[(2 \ell+1) \frac{v}{\mu}+(1-2 \lambda) \frac{\mu}{v}\right] \sqrt{\alpha} \frac{\partial}{\partial q}\right. \\
& \left.+\alpha\left(\varepsilon^{\prime}-2 \lambda-2 \lambda \ell\right)\right\} \zeta(q)=0 .
\end{aligned}
$$

Now we make a variable transformation by demanding $z=$ $2 \mu^{2}-1$, where the variable interval is $z \in(-1,1)$; then one can obtain

$$
\begin{aligned}
& \left\{\left(1-z^{2}\right) \frac{\partial^{2}}{\partial z^{2}}+[(\ell+1-\lambda)-(\ell+1+\lambda) z] \frac{\partial}{\partial z}\right. \\
& \left.+\frac{1}{4}\left(\varepsilon^{\prime}-2 \lambda-2 \lambda \ell\right)\right\} \zeta(z)=0 .
\end{aligned}
$$

It is important to point out that the wave function we used here will be regular at $z= \pm 1$ on the condition that $\zeta(z)$ is a polynomial, which is obtained by imposing the following constraint $(1 / 4)\left(\varepsilon^{\prime}-2 \lambda-2 \lambda \ell\right)=n(n+\ell+\lambda)$, with $n$ being a nonnegative integer. Then (27) turns into

$$
\begin{aligned}
& \left\{\left(1-z^{2}\right) \frac{\partial^{2}}{\partial z^{2}}+[(\ell+1-\lambda)-(\ell+1+\lambda) z] \frac{\partial}{\partial z}\right. \\
& \quad+n(n+\ell+\lambda)\} \zeta(z)=0,
\end{aligned}
$$

whose solution can be written in terms of Jacobi polynomials as $\zeta(z)=P_{n}^{(a, b)}(z)$, where $a=\lambda-1, b=\ell$. In this case, the energy eigenvalue of the system can be expressed as

$$
\begin{aligned}
\varepsilon^{\prime \prime}= & 2 \ell^{2}+4 n^{2}+4 n \ell \\
& +[4 n+2(\ell+1)]\left(1+\sqrt{1-k^{\prime}}\right)
\end{aligned}
$$

with $\varepsilon^{\prime \prime}=\left(E^{2}+2 m \omega \hbar c^{2}-m^{2} c^{4}\right) / \alpha m^{2} \omega^{2} \hbar^{2} c^{2}$.

Therefore the energy eigenvalues can be derived from (29) as

$$
\begin{aligned}
& E_{n \ell}= \pm m c^{2}\left\{\left(4 n^{2}+4 n \ell+2 \ell^{2}\right) \frac{\alpha \omega^{2} \hbar^{2}}{c^{2}}-\frac{2 \hbar \omega}{m c^{2}}-\frac{w^{2} \theta \ell}{c^{2}}+1\right. \\
& +\frac{2 \alpha \omega^{2} \hbar^{2}}{c^{2}}(2 n+\ell+1)[1 \\
& \left.\left.+\sqrt{1+\ell^{2}+\frac{\left(1+m^{2} w^{2} \theta^{2} / 4 \hbar^{2}\right)-2 m w \alpha \hbar-m^{2} w^{2} \theta \alpha \ell}{\alpha^{2} m^{2} w^{2} \hbar^{2}}}\right]\right\}^{1 / 2} \\
& n, \ell=0,1,2, \ldots ;
\end{aligned}
$$

furthermore, the wave function may be expressed by

$$
\begin{aligned}
\psi_{1}(P)= & N e^{i \ell \vartheta}\left(\frac{1}{1+\alpha P^{2}}\right)^{\lambda / 2}\left(\frac{\alpha P^{2}}{1+\alpha P^{2}}\right)^{\ell / 2} \\
& \cdot P_{n}^{(a, b)}\left(\frac{2 \alpha P^{2}}{1+\alpha P^{2}}-1\right), \quad n, \boldsymbol{\ell}=0,1,2, \ldots
\end{aligned}
$$


Then the wave function of the system is

$$
\psi=N\left(\begin{array}{c}
1 \\
\frac{E_{n \boldsymbol{\ell}}}{m c^{2}} \\
\frac{-\left[(\cos \vartheta+i(\boldsymbol{\theta} / 2 \hbar) m \omega \sin \vartheta) P+\hbar m w\left(1+\alpha P^{2}\right)(\cos \vartheta(\partial / \partial P)-((\sin \vartheta) / P)(\partial / \partial \vartheta))\right]}{m c} \\
\frac{-\left[(\sin \vartheta-i(\boldsymbol{\theta} / 2 \hbar) m w \cos \vartheta) P+m \omega \hbar\left(1+\alpha P^{2}\right)(\sin \vartheta(\partial / \partial P)+((\cos \vartheta) / P)(\partial / \partial \vartheta))\right]}{m c} \\
0
\end{array}\right) \psi_{1}(P, \vartheta) .
$$

Now the Jacobi polynomial [45] is employed to obtain the other components wave:

$$
\frac{d P_{n}^{(a, b)}(t)}{d t}=\frac{1}{2}(n+a+b+1) P_{n-1}^{(a+1, b+1)}(t) ;
$$

we finally have

$$
\begin{aligned}
& \psi_{2}(P, \vartheta)=\frac{E_{n \ell}}{m c^{2}} \psi_{1}(P, \vartheta)=N \frac{E_{n \ell}}{m c^{2}} e^{i \ell \vartheta}\left(\frac{1}{1+\alpha P^{2}}\right)^{\lambda / 2}\left(\frac{\alpha P^{2}}{1+\alpha P^{2}}\right)^{\ell / 2} P_{n}^{(a, b)}\left(\frac{\alpha P^{2}-1}{1+\alpha P^{2}}\right) \\
& \psi_{3}(P, \vartheta)=-\frac{\left[(\cos \vartheta+i(\boldsymbol{\theta} / 2 \hbar) m \omega \sin \vartheta) P+\hbar m w\left(1+\alpha P^{2}\right)(\cos \vartheta(\partial / \partial P)-((\sin \vartheta) / P)(\partial / \partial \vartheta))\right]}{m c} \psi_{1}(P, \vartheta) \\
& =-N e^{i \ell \vartheta}\left(\frac{1}{1+\alpha P^{2}}\right)^{\lambda / 2}\left(\frac{\alpha P^{2}}{1+\alpha P^{2}}\right)^{\ell / 2} \\
& \cdot \frac{1}{m c}\left\{\left[\left(\cos \vartheta+i \frac{\boldsymbol{\theta}}{2 \hbar} m w \sin \vartheta\right) P+\hbar m w \cos \vartheta\left(-\lambda \alpha P+\frac{\boldsymbol{\ell}}{P}\right)-i \hbar m w \boldsymbol{\ell}\left(1+\alpha P^{2}\right) \frac{\sin \vartheta}{P}\right] p_{n}^{(\lambda-1, \boldsymbol{\ell})}\right. \\
& \left.+\hbar m w \cos \vartheta \frac{2 \alpha P}{1+\alpha P^{2}}(n+\boldsymbol{\ell}+\lambda) p_{n-1}^{(\lambda, \boldsymbol{\ell}+1)}\right\}, \\
& \psi_{4}(P, \vartheta)=-\frac{\left[(\sin \vartheta-i(\boldsymbol{\theta} / 2 \hbar) m w \cos \vartheta) P+m \omega \hbar\left(1+\alpha P^{2}\right)(\sin \vartheta(\partial / \partial P)+((\cos \vartheta) / P)(\partial / \partial \vartheta))\right]}{m c} \psi_{1}(P, \vartheta) \\
& =-N e^{i \ell \vartheta}\left(\frac{1}{1+\alpha P^{2}}\right)^{\lambda / 2}\left(\frac{\alpha P^{2}}{1+\alpha P^{2}}\right)^{\ell / 2} \\
& \cdot \frac{1}{m c}\left\{\left[\left(\sin \vartheta-i \frac{\boldsymbol{\theta}}{2 \hbar} m w \cos \vartheta\right) P+m w \hbar \sin \vartheta\left(-\lambda \alpha P+\frac{\boldsymbol{\ell}}{P}\right)+i \hbar m w \boldsymbol{\ell}\left(1+\alpha P^{2}\right) \frac{\cos \vartheta}{P}\right] p_{n}^{(\lambda-1, \boldsymbol{\ell})}\right. \\
& \left.+\hbar m w \sin \vartheta \frac{2 \alpha P}{1+\alpha P^{2}}(n+\boldsymbol{\ell}+\lambda) p_{n-1}^{(\lambda, \boldsymbol{\ell}+1)}\right\}, \\
& \psi_{5}(P, \vartheta)=0 .
\end{aligned}
$$

After ending this part, we determine the normalization constant $N$ by demanding

$$
\int_{-\infty}^{+\infty} \frac{d^{2} p}{\left(1+\alpha P^{2}\right)} \bar{\psi}(P) \beta^{0} \psi(P)=1,
$$

besides, according to the property of the Jacobi polynomial,

$$
\begin{aligned}
& \int_{-1}^{+1} d t(1-t)^{a}(1+t)^{b}\left[P_{n}^{(a, b)}(t)\right]^{2} \\
& =\frac{2^{a+b+1} \Gamma(a+n+1) \Gamma(b+n+1)}{n !(a+b+1+2 n) \Gamma(a+b+n+1)}
\end{aligned}
$$


we obtain

$$
N=\alpha^{1 / 2}\left[\frac{m c^{2} n !(2 n+\lambda+\ell) \Gamma(n+\lambda+\ell)}{E_{n \ell} \Gamma(n+\lambda) \Gamma(n+\ell+1)}\right]^{1 / 2}
$$

then one can obtain the corresponding probability density of every component given by

$$
\begin{aligned}
& P^{\prime}{ }_{i}=\left|\int_{0}^{\pi} \int_{0}^{\infty} \bar{\psi}_{i}(P, \vartheta) \beta^{0} \psi_{i}(P, \vartheta) d p d \vartheta\right| \\
& i=1,2,3,4,5 .
\end{aligned}
$$

\section{The Problem under a Magnetic Field}

Now, in the presence of an external magnetic field, that is, $\vec{A}=\left(-B y^{(\mathrm{NC})} / 2 B x^{(\mathrm{NC})} / 20\right),(11)$ is transformed into

$$
\begin{aligned}
& {\left[\boldsymbol{\beta}^{0} \widetilde{E}-c \boldsymbol{\beta}^{1}\left(P_{x}^{(\mathrm{NC})}+\frac{e B y^{(\mathrm{NC})}}{2 c}-i m \boldsymbol{\omega} \boldsymbol{\eta}^{0} x^{(\mathrm{NC})}\right)\right.} \\
& \left.-c \boldsymbol{\beta}^{2}\left(P_{y}^{(\mathrm{NC})}-\frac{e B x^{(\mathrm{NC})}}{2 c}-i m \omega \boldsymbol{\eta}^{0} y^{(\mathrm{NC})}\right)-m c^{2}\right] \\
& \cdot \widetilde{\psi}=0
\end{aligned}
$$

here the spinor $\widetilde{\psi}$ is also a vector with five components which reads

$$
\widetilde{\psi}=\left(\widetilde{\psi}_{1}, \widetilde{\psi}_{2}, \widetilde{\psi}_{3}, \widetilde{\psi}_{4}, \widetilde{\psi}_{5}\right)^{T}
$$

Substituting $\widetilde{\psi}$ into (39) one can obtain

$$
\begin{aligned}
& -m c^{2} \widetilde{\psi}_{1}+\widetilde{E} \widetilde{\psi}_{2} \\
& +c\left(P_{x}^{(\mathrm{NC})}+\frac{e B y^{(\mathrm{NC})}}{2 c}+i m w x^{(\mathrm{NC})}\right) \widetilde{\psi}_{3} \\
& +c\left(P_{y}^{(\mathrm{NC})}-\frac{e B x^{(\mathrm{NC})}}{2 c}+i m w y^{(\mathrm{NC})}\right) \widetilde{\psi}_{4}=0
\end{aligned}
$$

$E \widetilde{\psi}_{1}-m c^{2} \widetilde{\psi}_{2}=0$

$$
c\left(P_{x}^{(\mathrm{NC})}+\frac{e B y^{(\mathrm{NC})}}{2 c}-i m w x^{(\mathrm{NC})}\right) \widetilde{\psi}_{1}+m c^{2} \widetilde{\psi}_{3}=0,
$$$$
c\left(P_{y}^{(\mathrm{NC})}-\frac{e B x^{(\mathrm{NC})}}{2 c}-i m w y^{(\mathrm{NC})}\right) \widetilde{\psi}_{1}+m c^{2} \widetilde{\psi}_{4}=0 \text {, }
$$

$m c^{2} \tilde{\psi}_{5}=0$.
Simplifying (41) gives

$$
\begin{aligned}
& \left\{\left[\left(1+\frac{e B \boldsymbol{\theta}}{4 \hbar c}\right) \widehat{P}_{x}+\frac{e B \widehat{y}}{2 c}+i m w\left(\widehat{x}-\frac{\boldsymbol{\theta}}{2 \hbar} \widehat{P}_{y}\right)\right]\right. \\
& \cdot\left[\left(1+\frac{e B \boldsymbol{\theta}}{4 \hbar c}\right) \widehat{P}_{x}+\frac{e B \widehat{y}}{2 c}-i m w\left(\widehat{x}-\frac{\boldsymbol{\theta}}{2 \hbar} \widehat{P}_{y}\right)\right] \\
& +\left[\left(1+\frac{e B \boldsymbol{\theta}}{4 \hbar c}\right) \widehat{P}_{y}-\frac{e B \widehat{x}}{2 c}+i m w\left(\widehat{y}+\frac{\boldsymbol{\theta}}{2 \hbar} \widehat{P}_{x}\right)\right] \\
& +\left[\left(1+\frac{e B \boldsymbol{\theta}}{4 \hbar c}\right) \widehat{P}_{y}-\frac{e B \widehat{x}}{2 c}-i m w\left(\widehat{y}+\frac{\boldsymbol{\theta}}{2 \hbar} \widehat{P}_{x}\right)\right] \\
& \left.+\frac{m^{2} c^{4}-\widetilde{E}^{2}}{c^{2}}\right\} \widetilde{\psi}_{1}=0,
\end{aligned}
$$

and considering (16) and (42) one can obtain

$$
\begin{aligned}
& \left\{\left\{\left(1+\frac{e B \boldsymbol{\theta}}{4 \hbar c}\right)^{2}+\frac{m^{2} w^{2} \boldsymbol{\theta}^{2}}{4 \hbar^{2}}-2 \hbar \alpha\left(m w\left(1+\frac{e B \boldsymbol{\theta}}{4 \hbar c}\right)\right.\right.\right. \\
& \left.+\frac{e B \boldsymbol{\theta} m w}{4 \hbar c}\right)-\alpha \hbar \boldsymbol{\ell}\left[\frac{e B}{c}\left(1+\frac{e B \boldsymbol{\theta}}{4 \hbar c}\right)+\frac{m^{2} w^{2} \boldsymbol{\theta}}{\hbar}\right. \\
& \left.\left.-2 \hbar \alpha \frac{e B m w}{c}\right]\right\} P^{2}+\left[m^{2} w^{2}+\left(\frac{e B}{2 c}\right)^{2}\right]\left(x^{2}+y^{2}\right) \\
& +\frac{m^{2} c^{4}-\widetilde{E}^{2}}{c^{2}}-2 \hbar\left[m w\left(1+\frac{e B \boldsymbol{\theta}}{4 \hbar c}\right)+\frac{e B \boldsymbol{\theta} m w}{4 \hbar c}\right] \\
& \left.-\hbar_{\boldsymbol{\ell}}\left[\frac{e B}{c}\left(1+\frac{e B \boldsymbol{\theta}}{4 \hbar c}\right)+\frac{m^{2} w^{2} \boldsymbol{\theta}}{\hbar}-2 \hbar \alpha \frac{e B m w}{c}\right]\right\} \\
& \cdot \widetilde{\psi}_{1}(P, \vartheta)=0 .
\end{aligned}
$$

Now, by a series of analogical algebraic operations and for the sake of simplification, we just give the following results:

The energy eigenvalues of the system are

$$
\begin{aligned}
\widetilde{E}_{\tilde{n} \ell} & = \pm m c^{2}\left\{\frac { \alpha \hbar ^ { 2 } } { m ^ { 2 } c ^ { 2 } } ( 4 \widetilde { n } ^ { 2 } + 4 \widetilde { n } \ell + 2 \ell ^ { 2 } ) \left[m^{2} w^{2}\right.\right. \\
& \left.+\left(\frac{e B}{2 c}\right)^{2}\right]-\frac{2 \hbar}{m^{2} c^{2}}\left[m w\left(1+\frac{e B \theta}{4 \hbar c}\right)+\frac{e B \theta m w}{4 \hbar c}\right] \\
& -\frac{\hbar \ell}{m^{2} c^{2}}\left[\frac{e B}{c}\left(1+\frac{e B \theta}{4 \hbar c}\right)+\frac{m^{2} w^{2} \theta}{\hbar}\right. \\
& \left.-\frac{2 \hbar \alpha e B m w}{c}\right]+1+\frac{2 \hbar^{2}}{m^{2} c^{2}}(2 \widetilde{n}+\ell+1)(\alpha \\
& \left.\left.+\sqrt{\alpha^{2}-\alpha^{2} \widetilde{K}}\right)\left(m^{2} w^{2}+\left(\frac{e B}{2 c}\right)^{2}\right)\right\}^{1 / 2},
\end{aligned}
$$

where 


$$
\begin{aligned}
\widetilde{K} & =-\boldsymbol{\ell}^{2} \\
& -\frac{\left\{(1+e B \boldsymbol{\theta} / 4 \hbar c)^{2}+m^{2} w^{2} \boldsymbol{\theta}^{2} / 4 \hbar^{2}-2 \hbar \alpha(m w(1+e B \boldsymbol{\theta} / 4 \hbar c)+e B \boldsymbol{\theta} m w / 4 \hbar c)-\alpha \hbar \boldsymbol{\ell}\left[(e B / c)(1+e B \boldsymbol{\theta} / 4 \hbar c)+m^{2} w^{2} \boldsymbol{\theta} / \hbar-2 \hbar \alpha e B m w / c\right]\right\}}{\alpha^{2} \hbar^{2}\left[m^{2} w^{2}+(e B / 2 c)^{2}\right]} .
\end{aligned}
$$

The wave function can be expressed as thus the wave function of the system is

$$
\begin{aligned}
& \widetilde{\psi}_{1}(P, \vartheta)= \widetilde{N} e^{i \ell \vartheta}\left(\frac{1}{1+\alpha P^{2}}\right)^{\lambda^{\prime} / 2}\left(\frac{\alpha P^{2}}{1+\alpha P^{2}}\right)^{\ell / 2} \\
& \cdot P_{\widetilde{n}}^{\left(a^{\prime}, b^{\prime}\right)}\left(\frac{2 \alpha P^{2}}{1+\alpha P^{2}}-1\right), \\
& \tilde{n}, \ell=0,1,2,3,4, \ldots ;
\end{aligned}
$$

$$
\tilde{\psi}=\tilde{N}\left(\begin{array}{c}
1 \\
\frac{E_{\tilde{n} \ell}}{m c^{2}} \\
-\frac{\left\{[(1+e B \theta / 4 \hbar c) \cos \vartheta+(i m \omega \theta / 2 \hbar) \sin \vartheta] P+\left(1+\alpha P^{2}\right)\left((i \hbar e B / 2 c)\left(\partial / \partial P_{y}\right)+\hbar m w\left(\partial / \partial P_{x}\right)\right)\right\}}{m c} \\
\frac{\left\{[(1+e B \theta / 4 \hbar c) \sin \vartheta-(i m \omega \theta / 2 \hbar) \cos \vartheta] P+\left(1+\alpha P^{2}\right)\left(-(i \hbar e B / 2 c)\left(\partial / \partial P_{x}\right)+\hbar m w \partial / \partial P_{y}\right)\right\}}{m c} \\
0
\end{array}\right) \tilde{\psi}_{1}(P,
$$

9) ;

then the other components' wave functions of the system are

$$
\begin{aligned}
& \widetilde{\psi}_{2}(P, \vartheta)=\frac{E_{\tilde{n} \ell}}{m c^{2}} \widetilde{\psi}_{1}(P, \vartheta)=\widetilde{N} \frac{E_{\tilde{n} \ell}}{m c^{2}} e^{i \ell \vartheta}\left(\frac{1}{1+\alpha P^{2}}\right)^{\lambda^{\prime} / 2}\left(\frac{\alpha P^{2}}{1+\alpha P^{2}}\right)^{\ell / 2} P_{\widetilde{n}}^{\left(a^{\prime}, b^{\prime}\right)}\left(\frac{2 \alpha P^{2}}{1+\alpha P^{2}}-1\right), \\
& \widetilde{\psi}_{3}(P, \vartheta)=-\frac{\left\{[(1+e B \boldsymbol{\theta} / 4 \hbar c) \cos \vartheta+(i m \omega \boldsymbol{\theta} / 2 \hbar) \sin \vartheta] P+\left(1+\alpha P^{2}\right)\left((i \hbar e B / 2 c)\left(\partial / \partial P_{y}\right)+\hbar m w\left(\partial / \partial P_{x}\right)\right)\right\}}{m c} \widetilde{\psi}_{1}(P, \vartheta) \\
& =-\frac{\widetilde{N} e^{i \ell \vartheta}}{m c}\left(\frac{1}{1+\alpha P^{2}}\right)^{\lambda^{\prime} / 2}\left(\frac{\alpha P^{2}}{1+\alpha P^{2}}\right)^{\ell / 2}\left\{\left\{\left[\left(1+\frac{e B \boldsymbol{\theta}}{4 \hbar c}\right) \cos \vartheta+\frac{i m \omega \boldsymbol{\theta}}{2 \hbar} \sin \vartheta\right] P\right.\right. \\
& \left.+\left(\frac{i \hbar e B}{2 c} \sin \vartheta+\hbar m w \cos \vartheta\right)\left(-\lambda^{\prime} \alpha P+\frac{\boldsymbol{\ell}}{P}\right)-\frac{\hbar e B \boldsymbol{\ell}}{2 c}\left(1+\alpha P^{2}\right) \frac{\cos \vartheta}{P}-i \boldsymbol{\ell} \hbar m w\left(1+\alpha P^{2}\right) \frac{\sin \vartheta}{P}\right\} P_{\widetilde{n}}^{\left(\lambda^{\prime}-1, \boldsymbol{\ell}\right)} \\
& \left.+\left(\frac{i \hbar e B}{2 c} \sin \vartheta+\hbar m w \cos \vartheta\right) \frac{2 \alpha P}{1+\alpha P^{2}}\left(n+\boldsymbol{\ell}+\lambda^{\prime}\right) P_{\widetilde{n}-1}^{\left(\lambda^{\prime}, \ell-1\right)}\right\}, \\
& \widetilde{\psi}_{4}(P, \vartheta)=-\frac{\left\{[(1+e B \boldsymbol{\theta} / 4 \hbar c) \sin \vartheta-(i m \omega \boldsymbol{\theta} / 2 \hbar) \cos \vartheta] P+\left(1+\alpha P^{2}\right)\left(-(i \hbar e B / 2 c)\left(\partial / \partial P_{x}\right)+\hbar m w\left(\partial / \partial P P_{y}\right)\right)\right\}}{m c} \widetilde{\psi}_{1}(P, \vartheta) \\
& =-\frac{\widetilde{N} e^{i \ell \vartheta}}{m c}\left(\frac{1}{1+\alpha P^{2}}\right)^{\lambda^{\prime} / 2}\left(\frac{\alpha P^{2}}{1+\alpha P^{2}}\right)^{\ell / 2}\left\{\left\{\left[\left(1+\frac{e B \boldsymbol{\theta}}{4 \hbar c}\right) \sin \vartheta-\frac{i m \omega \boldsymbol{\theta}}{2 \hbar} \cos \vartheta\right] P\right.\right. \\
& \left.+\left(-\frac{i \hbar e B}{2 c} \cos \vartheta+\hbar m w \sin \vartheta\right)\left(-\lambda \alpha P+\frac{\boldsymbol{\ell}}{P}\right)+\left(\frac{i \hbar e B}{2 c} \frac{\sin \vartheta}{P}+\hbar m w \frac{\cos \vartheta}{P}\right) i \boldsymbol{\ell}\left(1+\alpha P^{2}\right)\right\} P_{\widetilde{n}}^{\left(\lambda^{\prime}-1, \ell\right)}+\left(-\frac{i \hbar e B}{2 c} \cos \vartheta\right. \\
& \left.+\hbar m w \sin \vartheta) \frac{2 \alpha P}{1+\alpha P^{2}}\left(n+\boldsymbol{\ell}+\lambda^{\prime}\right) P_{\widetilde{n}-1}^{\left(\lambda^{\prime}, \boldsymbol{\ell}-1\right)}\right\}, \\
& \widetilde{\psi}_{5}(P, \vartheta)=0 \text {. }
\end{aligned}
$$


The normalization constant $N$ is

$$
\widetilde{N}=\alpha^{1 / 2}\left[\frac{M c^{2} \widetilde{n} !\left(2 \widetilde{n}+\lambda^{\prime}+\boldsymbol{\ell}\right) \Gamma\left(\tilde{n}+\lambda^{\prime}+\boldsymbol{\ell}\right)}{E_{\tilde{n} \boldsymbol{\ell}} \Gamma\left(\tilde{n}+\lambda^{\prime}\right) \Gamma(\tilde{n}+\boldsymbol{\ell}+1)}\right]^{1 / 2} ;
$$

finally, the corresponding probability density of every component can be expressed as

$$
\begin{aligned}
& P^{\prime \prime}=\left|\int_{0}^{\pi} \int_{0}^{\infty} \overline{\widetilde{\psi}}_{i}(P, \vartheta) \boldsymbol{\beta}^{0} \widetilde{\psi}_{i}(P, \vartheta) d p d \vartheta\right|, \\
& i=1,2,3,4,5 .
\end{aligned}
$$

\section{Special Cases and Discussions}

In this section, the natural unit ( $\hbar=c=m=w=1)$ is employed. Now, let us check the special cases. First, when the minimal length parameter $\alpha \rightarrow 0$, the energy relation (44) reduces to

$$
\begin{gathered}
\widetilde{E}_{\tilde{n} \ell}= \pm m c^{2}\left\{1-\frac{2 \hbar}{m^{2} c^{2}}\left[m w\left(1+\frac{e B \theta}{4 \hbar c}\right)+\frac{e B \theta m w}{4 \hbar c}\right]\right. \\
\left.-\frac{\hbar \ell}{m^{2} c^{2}}\left[\frac{e B}{c}\left(1+\frac{e B \theta}{4 \hbar c}\right)+\frac{m^{2} w^{2} \theta}{\hbar}\right]+\widetilde{K^{\prime}}\right\}^{1 / 2}, \\
\tilde{n}, \ell=0,1,2, \ldots,
\end{gathered}
$$

where

$$
\begin{aligned}
\widetilde{K^{\prime}}= & \frac{2 \hbar^{2}}{m^{2} c^{2}}(2 \widetilde{n}+\ell+1)\left(m^{2} w^{2}+\left(\frac{e B}{2 c}\right)^{2}\right) \\
& \cdot\left(l^{2}+\frac{(1+e B \theta / 4 \hbar c)^{2}+m^{2} w^{2} \theta^{2} / 4 \hbar^{2}}{\hbar^{2}\left(m^{2} w^{2}+(e B / 2 c)^{2}\right)}\right)^{1 / 2} .
\end{aligned}
$$

The energy has plotted the energy eigenvalues versus $B$ in Figure 1 . We see that the energy $E$ increases monotonically with the magnetic field parameter $B$ and the tendency of the spectrum can be observed for large numbers. It also shows that, for one principal quantum number, the energy $E$ increases with the increase of the azimuthal quantum number. The energy relation in the special case of $\theta=0$, that is, for vanishing NC parameter, gives

$$
\begin{aligned}
\widetilde{E}_{\tilde{n} \boldsymbol{\ell}} & = \pm m c^{2}\left\{\frac{\alpha \hbar^{2}}{m^{2} c^{2}}\left(4 \widetilde{n}^{2}+4 \tilde{n} \boldsymbol{\ell}+2 \boldsymbol{\ell}^{2}\right)\right. \\
& {\left[m^{2} w^{2}+\left(\frac{e B}{2 c}\right)^{2}\right]-\frac{2 \hbar m w}{m^{2} c^{2}} } \\
& -\frac{\hbar \boldsymbol{\ell}(e B-2 \hbar \alpha e B m w)}{m^{2} c^{3}}+1+\frac{2 \hbar^{2}}{m^{2} c^{2}}(2 \widetilde{n}+\boldsymbol{\ell}+1) \\
& \left.\cdot\left(m^{2} w^{2}+\left(\frac{e B}{2 c}\right)^{2}\right)\left(\alpha+\alpha \sqrt{1-\widetilde{K^{\prime \prime}}}\right)\right\}^{1 / 2},
\end{aligned}
$$

where

$$
\begin{aligned}
& \widetilde{K^{\prime \prime}} \\
& =\frac{1-2 \hbar \alpha m w-2 \hbar^{2} \alpha^{2} e B m w l / c-l^{2} \alpha^{2} \hbar^{2}\left(m^{2} w^{2}+(e B / 2 c)^{2}\right)}{\alpha^{2} \hbar^{2}\left[m^{2} w^{2}+(e B / 2 c)^{2}\right]} .
\end{aligned}
$$

From the result shown in Figure 2, we see that the energy eigenvalues also increase monotonically with the magnetic

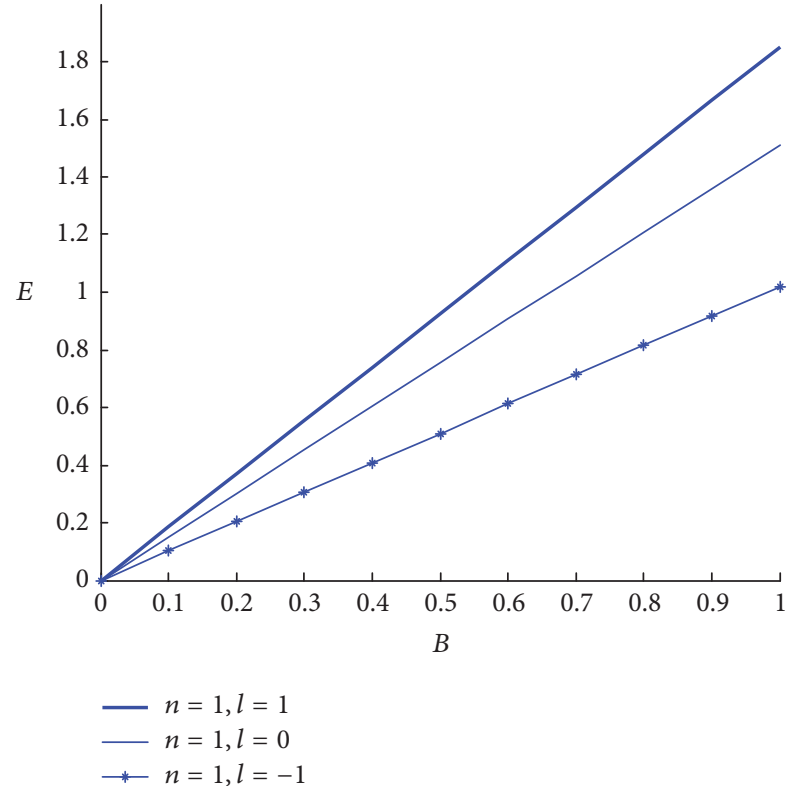

Figure 1: The energy eigenvalues versus $B(\theta=0.00003, m=1)$.

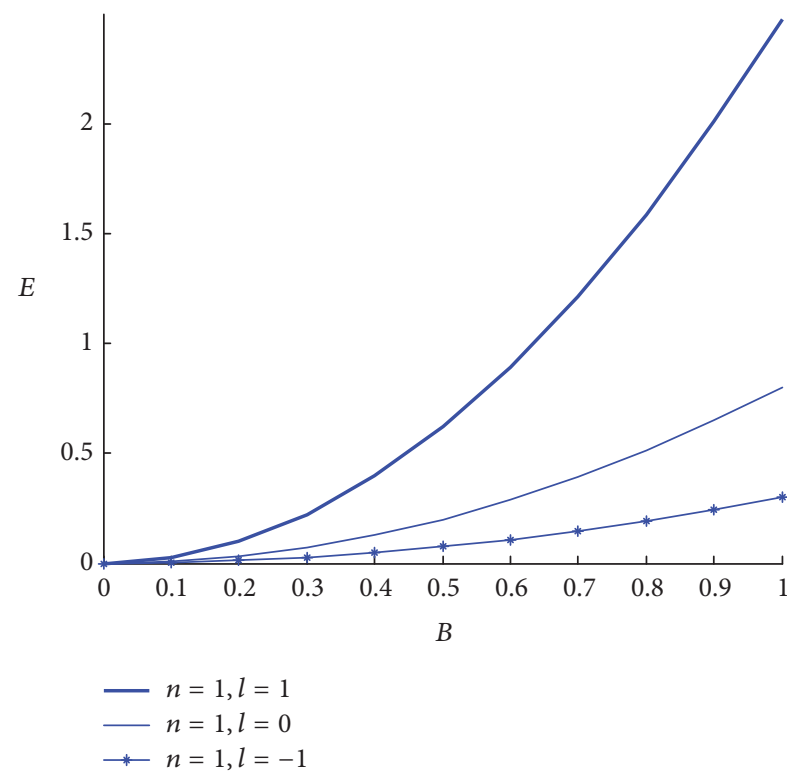

FIGURE 2: The energy eigenvalues versus $B(\alpha=0.05, m=1)$.

field variable $B$, and the profile shows that the energy eigenvalues first has a slow-growth and then rapidly increases. Finally, when both the noncommutative and minimal length parameters are absent, that is, $\alpha=\vartheta=0$, the energy spectrum degrades into

$$
\begin{aligned}
\widetilde{E}_{\tilde{n} \boldsymbol{\ell}} & = \pm m c^{2}\left[1-\frac{\hbar \boldsymbol{\ell} e B}{m^{2} c^{3}}-\frac{2 \hbar w}{m c^{2}}\right. \\
+ & \left.\frac{\hbar(4 \tilde{n}+2 \boldsymbol{\ell}+2)\left(w^{2}+(e B / 2 m c)^{2}\right)^{1 / 2}}{m c^{2}}\right]^{1 / 2} ;
\end{aligned}
$$




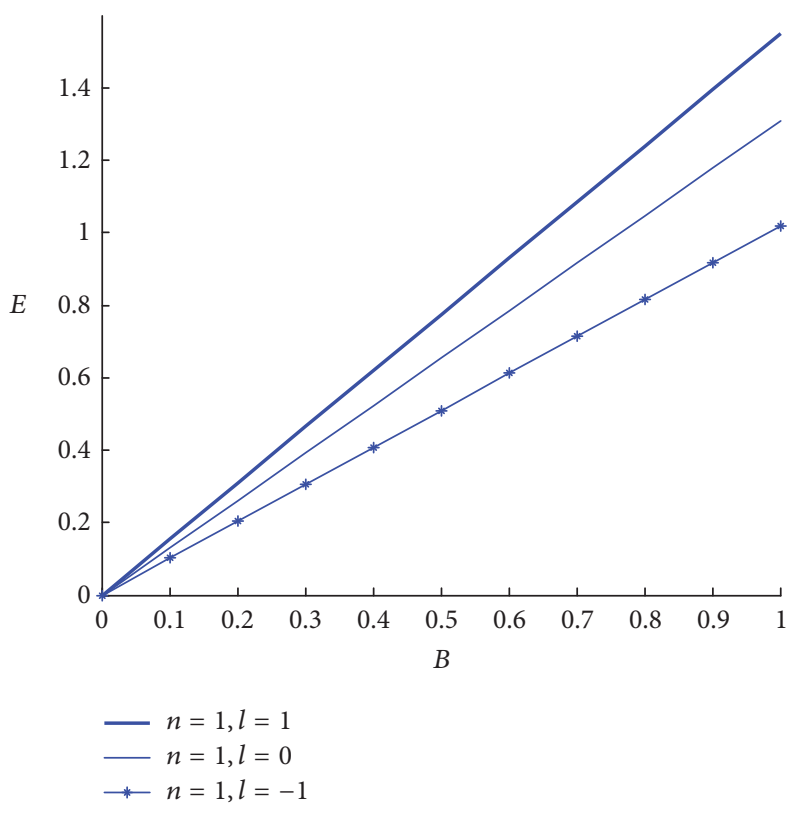

FIgure 3: The energy eigenvalues versus $B(m=1)$.

obviously, it is strictly consistent with [46]. In Figure 3, we have depicted the energy values versus $B$. It is also observed that the energy $E$ increases monotonically with the magnetic field parameter $B$, and for one principal quantum number, the energy $E$ increases with the increase of the azimuthal quantum number.

\section{Conclusions}

This paper is devoted to study of the $(2+1)$-dimensional Duffin-Kemmer-Petiau oscillator for spin 0 particle under a magnetic field in the presence of a minimal length in the NC space. We first analyze the DKP oscillator in the presence of a minimal length in NC space; by employing the momentum space representation, the energy spectrum is obtained as well as the wave functions and the corresponding probability densities of the system are reported in terms of the Jacobi polynomials. Subsequently, we generalize this quantum model into the framework of a magnetic field and report the corresponding results, respectively. Finally, this quantum model for special cases is discussed and the numerical results are depicted, respectively. It shows that the energy eigenvalues increase monotonically with the magnetic field variable $B$ for the minimal length parameter and the NC parameter, respectively, and for one principal quantum number, the energy $E$ increases with the increase of the azimuthal quantum number.

\section{Conflicts of Interest}

The authors declare that there are no conflicts of interest regarding the publication of this paper.

\section{Acknowledgments}

This work is supported by the National Natural Science Foundation of China (Grant nos. 11465006 and 11565009).

\section{References}

[1] H. Hassanabadi, Z. Molaee, and S. Zarrinkamar, "Noncommutative phase space schrödinger equation with minimal length," Advances in High Energy Physics, vol. 2014, Article ID 459345, 6 pages, 2014.

[2] A. Boumali and H. Hassanabadi, "Exact solutions of the $(2+1)-$ dimensional dirac oscillator under a magnetic field in the presence of a minimal length in the non-commutative phase space," Zeitschrift fur Naturforschung - Section A Journal of Physical Sciences, vol. 70, no. 8, pp. 619-627, 2015.

[3] P. Pedram, "Exact ultra cold neutrons' energy spectrum in gravitational quantum mechanics," The European Physical Journal C, vol. 73, no. 5, pp. 1-5, 2013.

[4] H. Hassanabadi, S. Zarrinkamar, and E. Maghsoodi, "Scattering states of woods-saxon interaction in minimal length quantum mechanics," Physics Letters. B. Particle Physics, Nuclear Physics and Cosmology, vol. 718, no. 5, pp. 678-682, 2012.

[5] D. Amati, M. Ciafaloni, and G. Veneziano, "Superstring collisions at planckian energies," Physics Letters B, vol. 197, no. 8, pp. 81-88, 1987.

[6] D. Amati, M. Ciafaloni, and G. Veneziano, "Can spacetime be probed below the string size?” Physics Letters B, vol. 216, no. 7, pp. 41-47, 1989.

[7] D. J. Gross and P. F. Mende, "The high-energy behavior of string scattering amplitudes," Physics Letters. B. Particle Physics, Nuclear Physics and Cosmology, vol. 197, no. 6, pp. 129-134, 1987.

[8] P. Pedram, "On the modification of the Hamiltonians' spectrum in gravitational quantum mechanics," Europhysics Letters, vol. 89, no. 5, Article ID 50008, pp. 50008-50013, 2010.

[9] P. Pedram, K. Nozari, and S. H. Taheri, "The effects of minimal length and maximal momentum on the transition rate of ultra cold neutrons in gravitational field," Journal of High Energy Physics, vol. 2011, article 93, 2011.

[10] K. Konishi, G. Paffuti, and P. Provero, "Minimum physical length and the generalized uncertainty principle in string theory," Physics Letters. B. Particle Physics, Nuclear Physics and Cosmology, vol. 234, no. 10, pp. 276-284, 1990.

[11] S. Benczik, L. N. Chang, D. Minic, and T. Takeuchi, "Hydrogenatom spectrum under a minimal-length hypothesis," Physical Review A-Atomic, Molecular, and Optical Physics, vol. 72, no. 1, Article ID 012104, pp. 573-573, 2005.

[12] M. Kato, "Particle theories with minimum observable length and open string theory," Physics Letters B, vol. 245, no. 5, pp. 43-47, 1990.

[13] S. Das, E. C. Vagenas, and A. F. Ali, "Discreteness of space from GUP II: relativistic wave equations," Physics Letters B: Nuclear, Elementary Particle and High-Energy Physics, vol. 690, no. 6, pp. 407-412, 2010.

[14] A. Kempf, G. Mangano, and R. B. Mann, "Hilbert space representation of the minimal length uncertainty relation," Physical Review. D. Third Series, vol. 52, no. 11, pp. 1108-1118, 1995.

[15] K. Nozari and A. Etemadi, "Minimal length, maximal momentum, and Hilbert space representation of quantum mechanics," Physical Review D, vol. 85, no. 11, pp. 502-512, 2012.

[16] A. Kempf, "Uncertainty relation in quantum mechanics with quantum group symmetry," Journal of Mathematical Physics, vol. 35, no. 14, pp. 4483-4496, 1994.

[17] H. Hinrichsen and A. Kempf, "Maximal localization in the presence of minimal uncertainties in positions and in momenta," Journal of Mathematical Physics, vol. 37, no. 17, pp. 2121-2137, 1996. 
[18] A. Kempf, "Non-pointlike particles in harmonic oscillators," Journal of Physics. A. Mathematical and General, vol. 30, no. 6, pp. 2093-2101, 1996.

[19] K. Nouicer, "Path integral for the harmonic oscillator in one dimension with nonzero minimum position uncertainty," Physics Letters. A, vol. 354, no. 7, pp. 399-405, 2006.

[20] K. Nozari and T. Azizi, "Gravitational induced uncertainty and dynamics of harmonic oscillator," General Relativity and Gravitation, vol. 38, no. 7, pp. 325-331, 2006.

[21] F. Brau and F. Buisseret, "Minimal length uncertainty relation and gravitational quantum well," Physical Review D, vol. 74, no. 1, Article ID 036002, pp. 307-307, 2006.

[22] M. M. Stetsko and V. M. Tkachuk, "Scattering problem in deformed space with minimal length," Physical Review A, vol. 76, no. 1, pp. 693-695, 2007.

[23] M. V. Battisti and G. Montani, "Quantum dynamics of the Taub universe in a generalized uncertainty principle framework," Physical Review D. Particles, Fields, Gravitation, and Cosmology, vol. 77, no. 6, pp. 179-184, 2008.

[24] M. V. Battisti and G. Montani, "The big-bang singularity in the framework of a generalized uncertainty principle," Physics Letters. B. Particle Physics, Nuclear Physics and Cosmology, vol. 656, no. 6, pp. 96-101, 2007.

[25] B. Majumder, "Effects of the modified uncertainty principle on the inflation parameters," Physics Letters, Section B: Nuclear, Elementary Particle and High-Energy Physics, vol. 709, no. 4, pp. 133-136, 2012.

[26] A. Tawfik and A. Diab, "Effect of the generalized uncertainty principle on compact stars," International Journal of Modern Physics. D. Gravitation, Astrophysics, Cosmology, vol. 23, no. 10, pp. 161-170, 2014.

[27] L.-Z. Wang, C.-Y. Long, and Z.-W. Long, "Quantization of space in the presence of a minimal length," Communications in Theoretical Physics, vol. 63, no. 6, pp. 709-714, 2015.

[28] B.-Q. Wang, C.-Y. Long, Z.-W. Long, and T. Xu, "Solutions of the Schrödinger equation under topological defects spacetimes and generalized uncertainty principle," European Physical Journal Plus, vol. 131, no. 10, pp. 378-388, 2016.

[29] H. S. Snyder, “Quantized space-time," Physical Review, vol. 71, no. 1, pp. 38-41, 1947.

[30] T. Banks, W. Fischler, S. H. Shenker, and L. Susskind, "M theory as a matrix model: a conjecture," Physical Review. D. Third Series, vol. 55, no. 8, pp. 5112-5128, 1997.

[31] A. E. Djemai, "Noncommutative classical mechanics," International Journal of Theoretical Physics, vol. 43, no. 2, pp. 299-314, 2004.

[32] J. Gamboa, M. Loewe, and J. C. Rojas, "Noncommutative quantum mechanics," Physical Review. D. Third Series, vol. 64, no. 6, Article ID 067901, 2001.

[33] J. M. Romero, J. A. Santiago, and J. D. Vergara, "Newton's second law in a non-commutative space," Physics Letters. A, vol. 310, no. 1, pp. 9-12, 2003.

[34] M. Daszkiewicz and C. J. Walczyk, "Newton equation for canonical, lie-algebraic, and quadratic deformation of classical space," Physical Review D. Particles, Fields, Gravitation, and Cosmology, vol. 77, no. 10, Article ID 105008, 2008.

[35] J. Jing, F. H. Liu, and J. F. Chen, "Classical and quantum mechanics in the generalized non-commutative plane," $E P L$, vol. 84, no. 6, Article ID 61001, 2008.

[36] B. Muthukumar and P. Mitra, "Noncommutative oscillators and the commutative limit," Physical Review. D. Third Series, vol. 66, no. 2, Article ID 027701, 3 pages, 2002.
[37] A. Kijanka and P. Kosinski, "Noncommutative isotropic harmonic oscillator," Physical Review D, vol. 70, no. 12, Article ID 127702, 2004.

[38] J. Jing, S.-H. Zhao, J.-F. Chen, and Z.-W. Long, "On the spectra of noncommutative 2D harmonic oscillator," The European Physical Journal C. Particles and Fields, vol. 54, no. 4, pp. 685690, 2008.

[39] A. Das, H. Falomir, M. Nieto, J. Gamboa, and F. Méndez, "Aharonov-bohm effect in a class of noncommutative theories," Physical Review D, vol. 84, no. 4, Article ID 045002, 2011.

[40] Z. Wang, Z.-W. Long, C.-Y. Long, and W. Zhang, "On the thermodynamic properties of the spinless duffin-kemmer-petiau oscillator in noncommutative plane," Advances in High Energy Physics, vol. 2015, 9 pages, 2015.

[41] Y. Nedjadi and R. C. Barrett, "The Duffin-Kemmer-Petiau oscillator," Journal of Physics. A. Mathematical and General, vol. 27, no. 12, pp. 4301-4315, 1994.

[42] Y. Nedjadi and R. C. Barrett, "On the properties of the DuffinKemmer-Petiau equation," Journal of Physics G: Nuclear and Particle Physics, vol. 19, no. 1, pp. 87-98, 1993.

[43] H. Hassanabadi, B. H. Yazarloo, S. Zarrinkamar, and A. A. Rajabi, "Duffin-Kemmer-Petiau equation under a scalar Coulomb interaction," Physical Review C, vol. 84, no. 6, Article ID 064003, 2011.

[44] L. B. Castro and L. P. de Oliveira, "Remarks on the spin-one duffin-kemmer-petiau equation in the presence of nonminimal vector interactions in $(3+1)$ dimensions," Advances in High Energy Physics, vol. 2014, 8 pages, 2014.

[45] I. S. Gradshteyn and I. M. Ryzhik, Tables of Integrals, Series and Products Academic, New York, 1980.

[46] A. Boumali, L. Chetouani, and H. Hassanabadi, "Two-dimensional Duffin-Kemmer-Petiau oscillator under an external magnetic field," Canadian Journal of Physics, vol. 91, no. 1, pp. 1-11, 2013. 

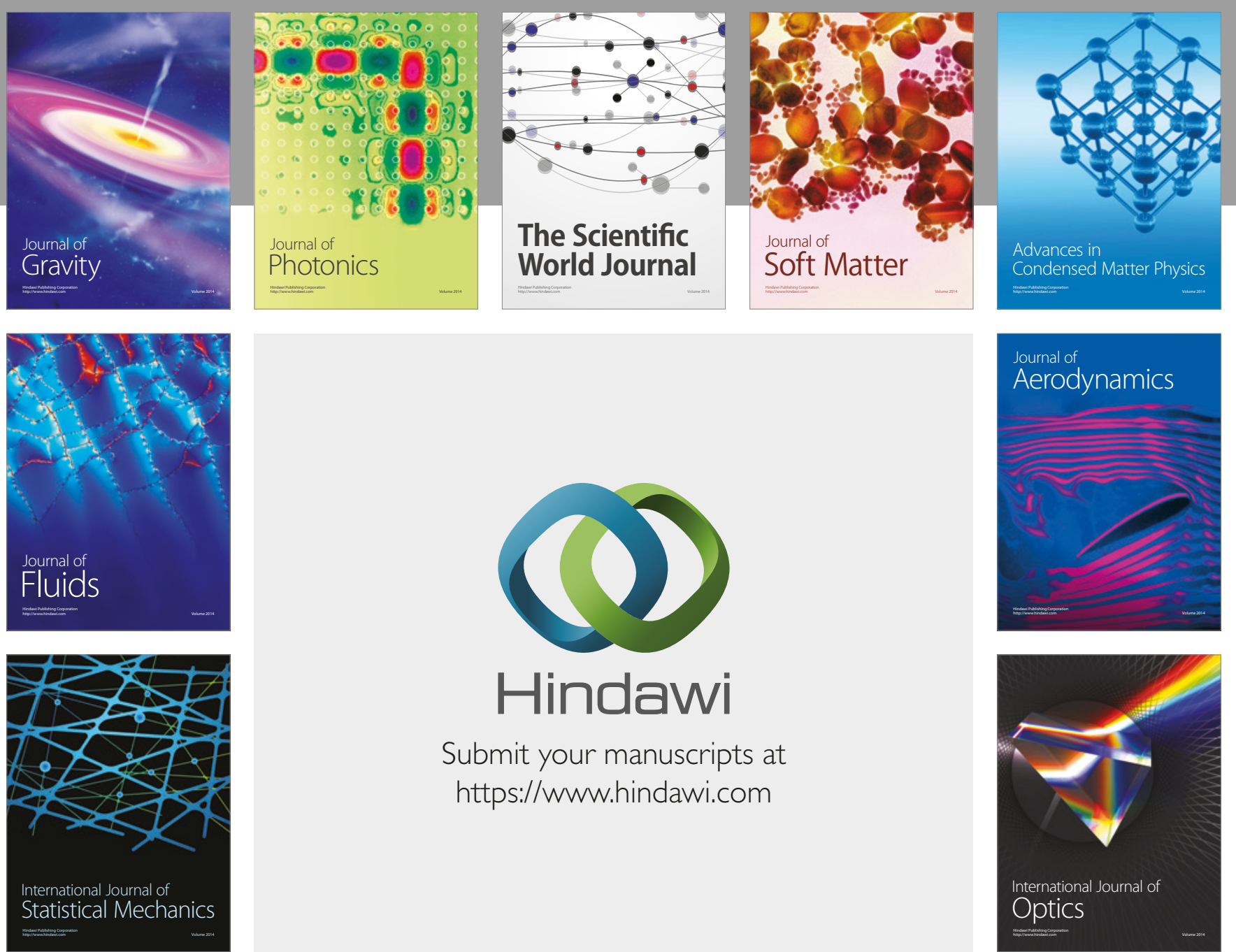

Submit your manuscripts at

https://www.hindawi.com
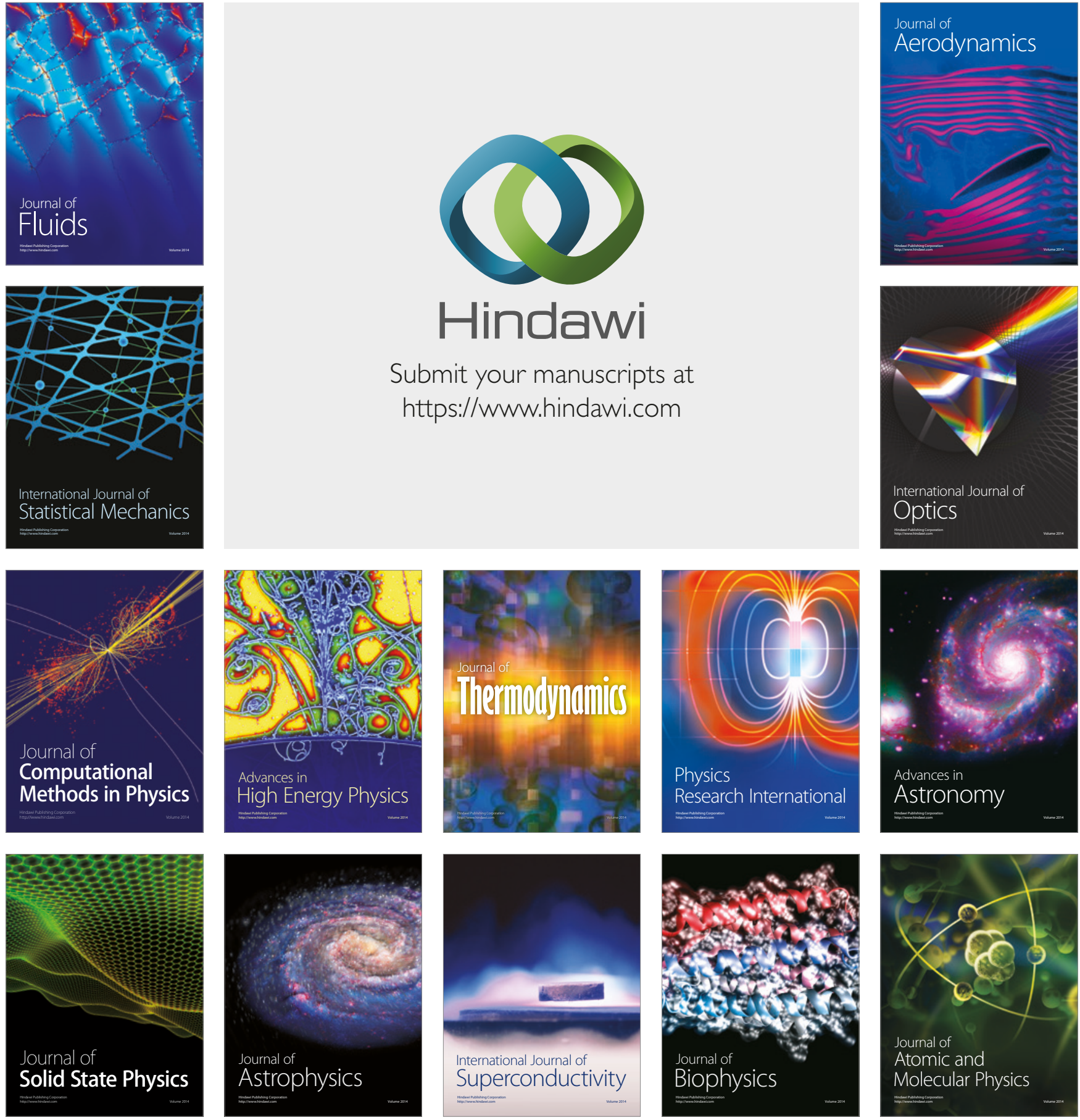